\title{
Electron mobility and Monte Carlo device simulation of MOSFETS
}

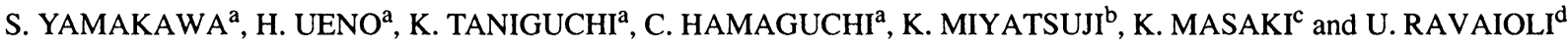 \\ ${ }^{\mathrm{a}}$ Department of Electronic Engineering Faculty of Engineering, Osaka University Suita City, Osaka 565, Japan, ${ }^{\mathrm{b}}$ Electronics Research \\ Laboratory Matsushita Electronics Corporation Takatsuki City, Osaka 569, Japan, ${ }^{\mathrm{C}}$ Anan College of Technology Anan City, Tokushima 774, \\ Japan, ${ }^{\mathrm{d}}$ Department of Electrical and Computer Engineering University of Illinois at Urbana-Champaign, Urbana IL 61801
}

\begin{abstract}
The electron mobility in the inversion layer of a MOSFET, formed on the (100) silicon surface, is calculated by using a Monte Carlo approach which takes into account size quantization, acoustic phonon scattering, intervalley phonon scattering and surface roughness scattering. Degeneracy is also considered because it is important at higher normal effective fields (high gate voltages). The main emphasis is placed on the influence of the specific autocovariance function, used to describe the surface roughness, on the electron mobility. It is found that the electron mobility calculated with roughness scattering rates based on the exponential function shows a good agreement with experiments. Device simulation of a MOSFET is carried out to demonstrate the usefulness of the present model, where $3 \mathrm{D}$ electron states are taken into account in addtion to the $2 \mathrm{D}$ electron states.
\end{abstract}

\section{INTRODUCTION}

A very complete MOSFET simulation was made by Fischetti and Laux[1], where they took into account size quantization and detailed models of the various scattering rates, including surface roughness scattering. Their calculated results give a good interpretation of the observed transport properties of hot electrons in MOSFETs. From these results it is evident that quantization of the electrons and the interface roughness scattering play an important role in the electron transport in MOSFETs. It should be pointed out that Monte Carlo simulations based on the three-dimensional motion of electrons in MOSFETs have to assume an unrealistic scattering of electrons at the interface. When electron quantization is taken into account, scattering of the electrons confined in a narrow region near the interface is well treated by introducing the interface roughness scattering. In this paper we are mainly concerned with the effect of interface roughness scattering on the electron mobility in MOSFETs. We also present a result of Monte Carlo device simulation of a MOSFET using the present model for electron scattering, where $2 \mathrm{D}$ and $3 \mathrm{D}$ electrons are taken into account.

\section{THEORETICAL MODELS}

\section{Phonon Scatterings}

The conduction band of $\mathrm{Si}$ has six valleys along the $<100>$ directions of the Brillouin zone. In MOSFET structures fabricated on (001) substrates, electrons are quantized due to a strong gate field and the six valleys result in two different series of subbands, classified by the effective masses of the valleys in the direction normal to the substrate: the subband series $E_{i}$ associ- 
ated with the two valleys with $m_{l}$ (longitudinal effective mass) perpendicular to the surface; and the subband series $E_{i}^{\prime}$ associated with the four valleys with $m_{t}$ (transverse effective mass) perpendicular to the surface.[2] The electronic states of 2DEG are calculated by solving the Schrödinger and Poisson equations self-consistently[2]

$$
\begin{gathered}
-\frac{\hbar^{2}}{2 m_{3}} \frac{\mathrm{d}^{2}}{\mathrm{~d} z^{2}} \zeta_{i}(z)-e \phi(z) \zeta_{i}(z)=E_{i} \zeta_{i}(z), \\
\frac{\mathrm{d}^{2} \phi}{\mathrm{d} z^{2}}=-\frac{1}{\kappa_{\mathrm{Si}} \varepsilon_{0}}\left[\rho_{\mathrm{depl}}(z)-e \sum_{i} N_{i} \zeta_{i}^{2}(z)\right],
\end{gathered}
$$

where $m_{3}$ is the effective mass perpendicular to the surface. In terms of the notation in Stern's paper, $E_{i}$ and $E_{i}^{\prime}$ are the subband energies with $m_{3}=m_{l}$ (2 degeneracy) and $m_{3}=m_{t}$ (4 degeneracy), respectively.[2] $\phi(z)$ is the electrostatic potential, $\rho_{\text {depl }}$ the charge density of the depletion layer, $\kappa_{\mathrm{Si}} \varepsilon_{0}$ the dielectric constant of $\mathrm{Si}$, and $N_{s}$ the electron sheet density in the inversion layer. Price[3] and Ridley[4], independently, reported an adequate approach to treat the scattering rates, where the three-dimensional nature of the phonons in the inversion layer is assumed. Under this assumption the derivation of the scattering rates for electron-phonon interaction is straightforward. In our simulation, we take into account acoustic phonon (in this paper the anisotropy of deformation potential interaction is not taken into account for simplicity) and intervalley phonon scattering except interface roughness scattering.

\section{Interface Roughness Scatterings}

Analysis of the electron mobility in Si-MOSFETs is often done by assuming a Gaussian autocovariance function.[5] However, more recent investigations indicate that the roughness may be better expressed by an exponential autocovariance function.[6]

The scattering rate for Gaussian function is written as

$$
\frac{1}{\tau_{G}(\boldsymbol{k} / /)}=\frac{m_{d} e^{2} E_{\mathrm{eff}}^{2} \Delta^{2} L^{2}}{2 \hbar^{3}} \int_{0}^{2 \pi} e^{-q^{2} L^{2} / 4}(1-\cos \theta) \mathrm{d} \theta .
$$

When the screening effect is included, the scattering rate for the exponential autocovariance function is then given by

$$
\begin{aligned}
\frac{1}{\tau_{E}\left(\boldsymbol{k}_{/ /}\right)}=\frac{m_{0} e^{2} E_{\mathrm{eff}}^{2} \Delta^{2} L^{2}}{2 \hbar^{3} \varepsilon(q)} & \\
& \int_{0}^{2 \pi} \frac{1}{\left(1+L^{2} q^{2} / 2\right)}(1-\cos \theta) \mathrm{d} \theta
\end{aligned}
$$

and similar expression may be obtained for the Gaussian autocovariance function, where $\varepsilon(q)$ is the dielectric function with screening effect and given by

$$
\varepsilon(q)=1+\frac{e^{2}}{2 \kappa_{\mathrm{Si}} \varepsilon_{0}} \frac{1}{q} \frac{m_{d}}{\pi \hbar^{2}} F(q),
$$

where

$$
F(q)=\sum_{v} \int \mathrm{d} z \int \mathrm{d} z^{\prime}\left|\zeta_{v}(z)\right|^{2}\left|\zeta_{v}\left(z^{\prime}\right)\right|^{2} e^{\left(-q\left|z-z^{\prime}\right|\right)} .
$$

\section{CALCULATIONS AND DISCUSSION}

We consider here electron transport in an n-type inversion layer formed at a (100) surface of MOSFET, at room temperature. In order to clarify the effect of surface roughness scattering, we calculate the electron mobility limited by the phonon scattering and then the mobility with the roughness scattering. In Fig.1 we show the calculated results of phononlimited mobility (solid circles), and mobility calcu-

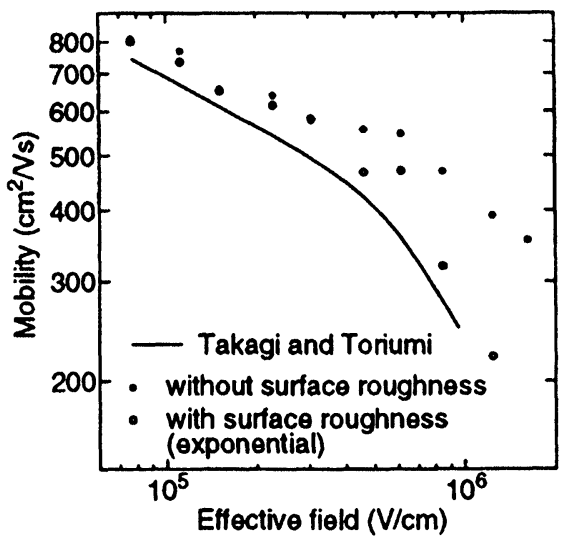

FIGURE 1 Electron mobility obtained from Monte Carlo simulation with (open circles) and without (solid circles) surface roughness scattering 
lated by taking into account of the surface roughness scattering where the exponential autocovariance function for the roughness is assumed and the degeneracy of carriers is considered (open circles). It is obvious from Fig. 1 that the phonon-limited mobility is much higher than the experimental one (solid line) and that the deviation is larger at higher normal effective fields. As expected, we find that the calculated results with the surface roughness, with $L=22 \AA$ and $\Delta=1.78 \AA$, and the degeneracy effect gives a better fit to the experimental data at higher effective fields.

We carried out Monte Carlo simulation of a SiMOSFET using the present model for the carrier scattering. In the calculation of electron mobility presented above the inversion layer is treated as uniform between source and drain. In the real device, however, the inversion layer is nonuniform and the electrons near the drain contact should be treated as threedimensional. On the other hand, the simulators based on three-dimensional electrons cannot treat surface roughness scattering theoretically. We, therefore, treat electrons near the source region as two-dimensional and near the drain region as three-dimensional. Figure 2 shows the device model used for the Monte Carlo simulation, where the gate length is $0.4 \mu \mathrm{m}$, the thickness of $\mathrm{SiO}_{2}$ is $100 \AA$, and the substrate doping is $N_{A}=5 \times 10^{16} \mathrm{~cm}^{-3}$. We treat electrons in the inversion layer region as two-dimensional. The high energy electrons, however, should be treated as threedimensional rather than as two-dimensional. In the initial condition for the device simulation we take 20,000 electrons in the source and drain region and

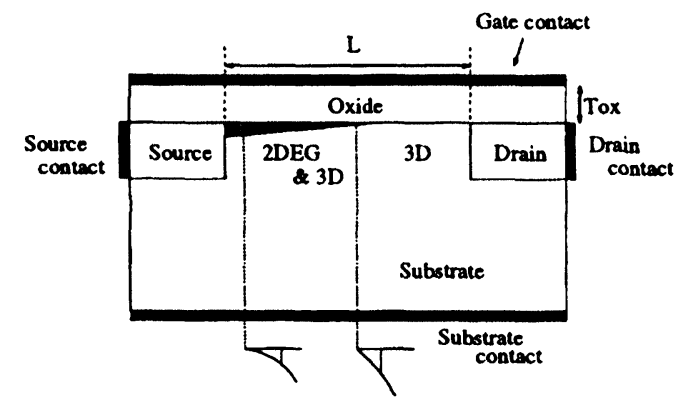

FIGURE 2 Si-MOSFET device structure used for the simulation

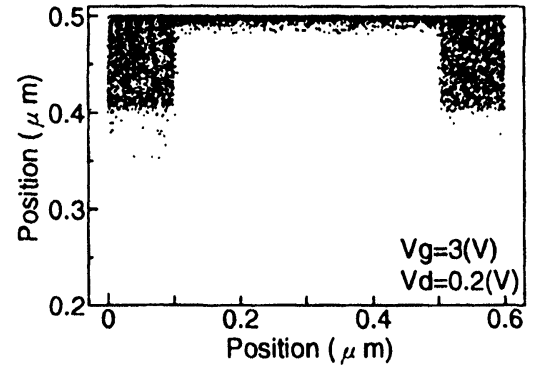

FIGURE 3 Electron distribution in the Si-MOSFET for gate voltage $3 \mathrm{~V}$ and drain voltage $0.2 \mathrm{~V}$

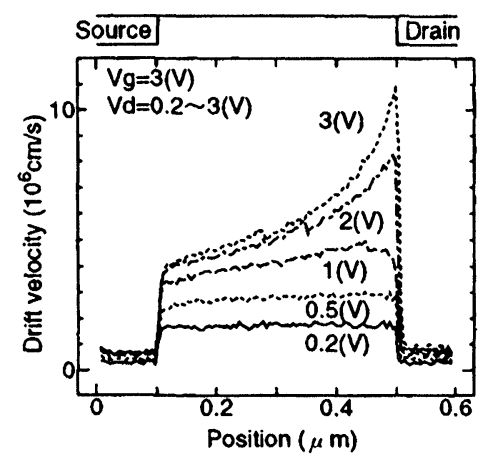

FIGURE 4 Position dependence of electron drift velocity. Gate voltage is $3 \mathrm{~V}$

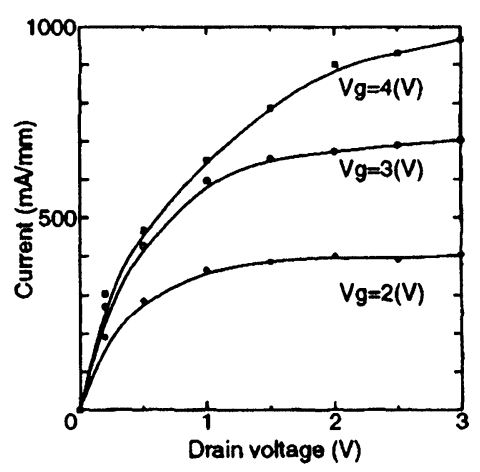

FIGURE $5 I_{D}$ versus $V_{D}$ characteristics of the Si-MOSFET obtained by Monte Carlo simulation

the simulation time is 30 ps. Figure 3 shows the electron distribution obtained by the present simulation. The gate voltage is $3 \mathrm{~V}$ and drain voltage is $0.2 \mathrm{~V}$. Figure 4 shows the position dependence of electron drift velocity. When drain bias is low, the drift velocity between source and drain is flat and increases in proportion to the field parallel to the surface. As drain voltage becomes high, velocity saturation can be 
observed near the drain region. Drain current versus drain voltage characteristics of the MOSFET are shown in Fig.5 for gate voltage $V_{G}=2,3$ and $4 \mathrm{~V}$. Although the present method assumes a very simple model for Monte Carlo device simulation, the obtained results show a good agreement with the experimental data in short channel MOSFETs.

\section{References}

[1] M. V. Fischetti and S. E. Laux, "Monte Carlo study of electron transport in silicon inversion layers," Phys. Rev. B48, 2244 (1993).
[2] F. Stern, "Self-consistent results for n-type Si inversion layers," Phys. Rev. B5, 4891 (1972).

[3] P. J. Price, "Two-dimensional electron transport in semiconductor layers, I. Phonon scattering," Ann. Phys. 133, 217 (1981).

[4] B. K. Ridley, "The electron-phonon interaction in quasi-twodimensional semiconductor quantum-well structure," J. Phys. C15, 5899 (1982).

[5] T. Ando, A. B. Fowler and F. Stern, "Electronic properties of two-dimensional systems," Rev. Mod. Phys. 54, 437 (1982).

[6] S. M. Goodnick, D. K. Ferry, C. W. Wilmsen, Z. Liliental, D. Fathy and O. L. Krivanek, "Surface roughness at the $\mathrm{Si}(100)$ $\mathrm{SiO}_{2}$ interface," Phys. Rev. B32, 8171 (1985).

[7] S. Takagi and A. Toriumi, "New experimental findings on hot carrier transport under velocity saturation regime in $\mathrm{Si}$ MOSFET," IEDM Tech. Dig., 711 (1992). 

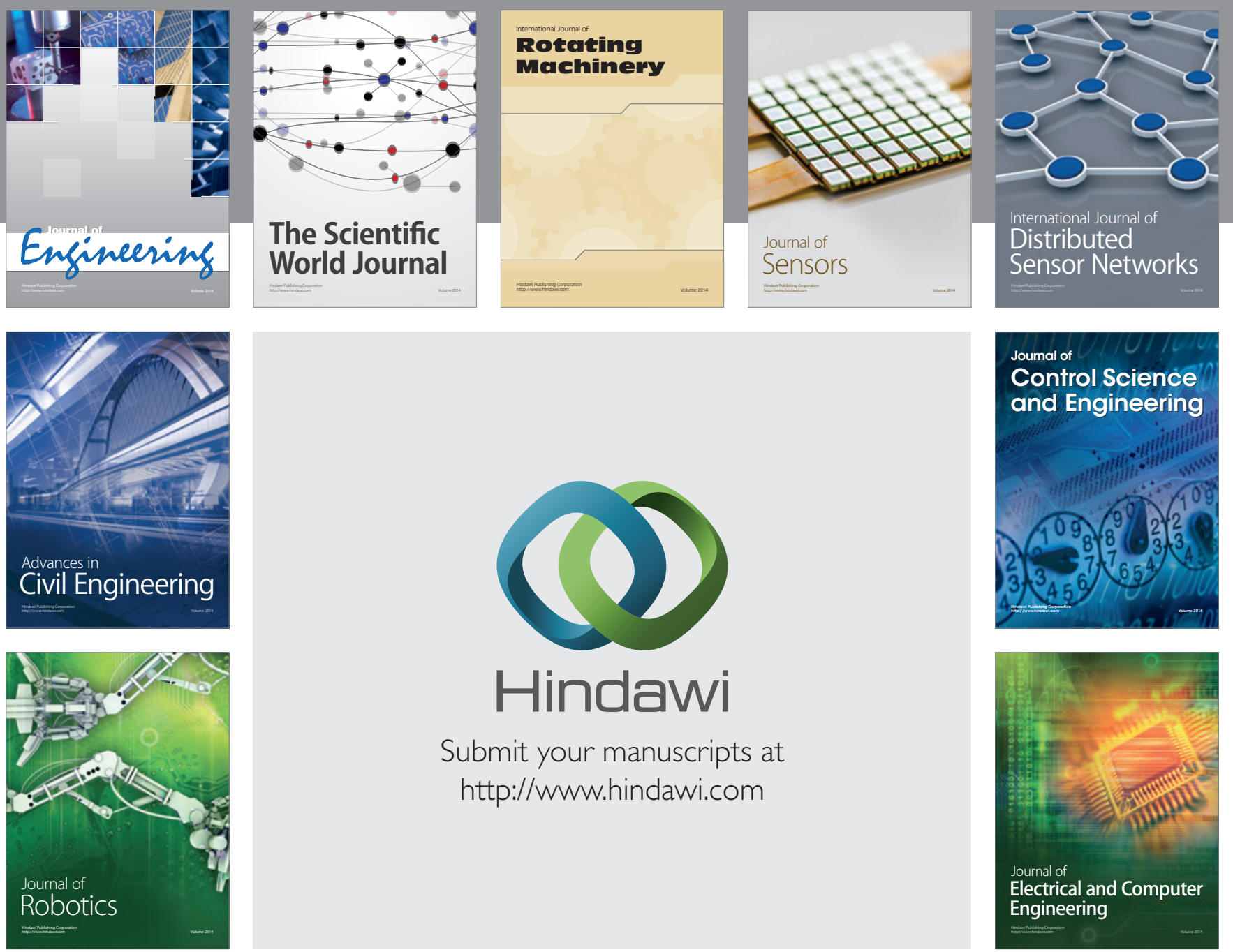

Submit your manuscripts at

http://www.hindawi.com
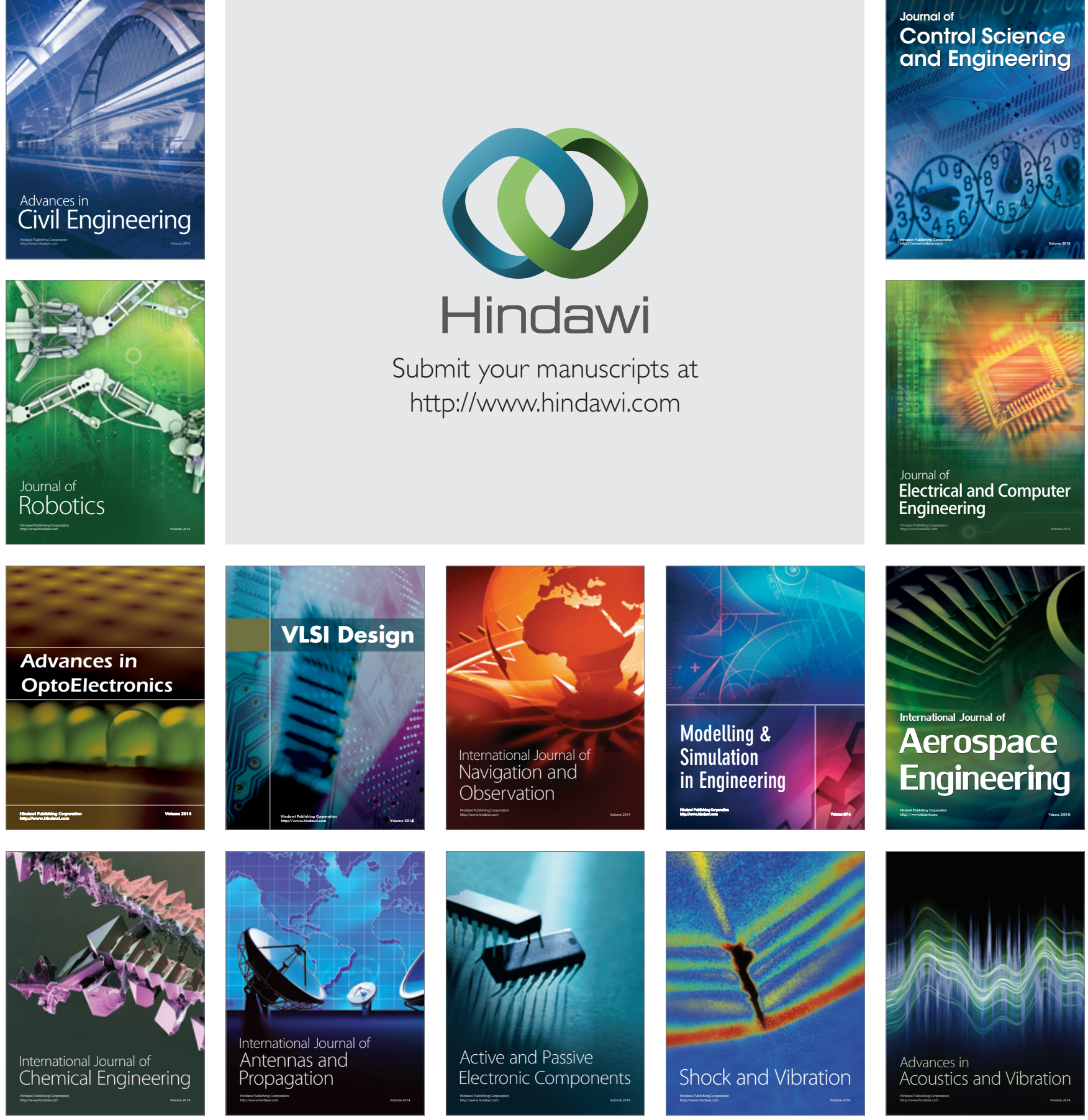